Vol. 111 (1988), 115-129

\title{
THE DIMENSION FORMULA OF THE SPACE OF CUSP FORMS OF WEIGHT ONE FOR $\Gamma_{0}(p)$
}

\author{
YOSHIO TANIGAWA AND HIROFUMI ISHIKAWA
}

\section{Introduction}

The purpose of this paper is to study the dimension formula for cusp forms of weight one, following the series of Hiramatsu [2] and HiramatsuAkiyama [3]. We define as usual the subgroup $\Gamma_{0}(N)$ of $S L_{2}(Z)$ by

$$
\Gamma_{0}(N)=\left\{\left(\begin{array}{ll}
a & b \\
c & d
\end{array}\right) \in S L(2, Z) \mid c \equiv 0(\bmod N)\right\} .
$$

In this paper we consider the case of a prime level, so we always put $\Gamma=\Gamma_{0}(p)$ for a prime number $p$. In Section 1, we define the Eisenstein series and determine the constant term matrix explicitly. In Section 2, we calculate the trace of certain invariant integral operator by the method of Selberg. First we define a Selberg type zeta function $z(\delta, \chi)$ in (2.4). It appears in the trace formula from the hyperbolic conjugacy classes. After analytic continuation, we take the residue of $z(\delta, \chi)$ at 0 and get

$$
\operatorname{dim} S_{1}(\Gamma, \chi)=\frac{1}{4} \operatorname{Res}_{\delta=0} z(\delta, \chi) .
$$

On the other hand, Deligne-Serre (c.f. Serre [8]) proved that this dimension is equal to the number of two dimensional Galois representations satisfying certain conditions. Thus the residue of the Selberg type zeta function contains an information of the number of such representations.

The main result of this paper was obtained by both authors independently. The first author would like to express his hearty thanks to Professor Hiramatsu and Dr. Akiyama for fruitful discussions.

\section{$\S 1$. Eisenstein series}

Let $k$ be a positive integer and $\chi$ a Dirichlet character modulo $p$. We

Received December 10, 1986. 
assume that $\chi(-1)=(-1)^{k}$. For $\sigma=\left(\begin{array}{ll}a & b \\ c & d\end{array}\right) \in \Gamma$, we put $\chi(\sigma)=\chi(d)$ and regard $\chi$ as a character of $\Gamma$. The discrete subgroup $\Gamma$ has two cusps represented by $\infty$ and 0 . Let $\Gamma_{\kappa}$ be the stabilizer of a cusp $\kappa$. We put

$$
\sigma_{\infty}=I \quad \text { and } \quad \sigma_{0}=\frac{1}{\sqrt{p}}\left(\begin{array}{rr}
0 & -1 \\
p & 0
\end{array}\right)
$$

where $I$ is the unit matrix. Then it holds that $\sigma_{\kappa} \infty=\kappa$ and $\sigma_{\kappa}^{-1} \Gamma_{\kappa} \sigma_{x}=\Gamma_{\infty}$.

Let $S$ be the upper half plane. For $z=x+\sqrt{-1} y \in S$, we denote the imaginary part of $z$ by $y(z)$. We define an automorphic factor of weight $k$ as

$$
j_{k}(\sigma, z)=\left(\frac{c z+d}{|c z+d|}\right)^{k}
$$

The Eisenstein series at a cusp $\kappa$ is defined by

$$
E_{\kappa}(z, s, k, \chi)=\sum_{\sigma \in \Gamma_{k} \backslash \Gamma} \chi(\sigma)^{-1} j_{k}\left(\sigma_{\kappa}^{-1} \sigma, z\right)^{-1} y\left(\sigma_{\kappa}^{-1} \sigma z\right)^{s},
$$

where $z \in S$ and $s \in C$. This series converges absolutely and uniformly for $z$ in any compact subset of $S$ and $s$ in any compact subset of $\{s \mid \operatorname{Re}(s)>1\}$. We put

$$
\begin{aligned}
E_{\kappa, \mu}(z, s, k, \chi) & =j_{k}\left(\sigma_{\mu}, z\right)^{-1} E_{\kappa}\left(\sigma_{\mu} z, s, k, \chi\right) \\
& =\sum_{\sigma \in \Gamma_{\boldsymbol{k}} \backslash \Gamma} \chi(\sigma)^{-1} j_{k}\left(\sigma_{\kappa}^{-1} \sigma \sigma_{\mu}, z\right)^{-1} y\left(\sigma_{\kappa}^{-1} \sigma \sigma_{\mu} z\right)^{s}
\end{aligned}
$$

for any pair of cusps $\kappa, \mu$. We often omit the weight $k$ and the character $\chi$ if there is no fear of confusion. Since $E_{\kappa, \mu}(z, s)$ is a periodic function in $z$ with period 1 , we have a Fourier series expansion

$$
E_{\kappa, \mu}(z, s)=\sum_{m \in \boldsymbol{Z}} a_{\kappa, \mu}(m ; y, s) e(m x)
$$

with

$$
a_{\kappa, \mu}(m ; y, s)=\int_{0}^{1} E_{\kappa, \mu}(z, s) e(-m x) d x
$$

where $e(x)=\exp (2 \pi \sqrt{-1} x)$. The constant term of $E_{\kappa, \mu}(z, s)$ has the following form

$$
a_{\kappa, \mu}(0 ; y, s)=\delta_{\kappa, \mu} y^{s}+m_{\kappa, \mu}(s, k, \chi) y^{1-s}
$$

with Kronecker's $\delta$. The matrix $M(s, k, \chi)=\left(m_{\kappa, \mu}(s, k, \chi)\right)$ appearing in the 
constant term of $E_{\kappa, \mu}(z, s)$, which is called the constant term matrix, plays an important role in the theory of Eisenstein series (cf. Kubota [6]). We will determine $M(s, k, \chi)$ explicitly in the following Proposition.

Proposition 1. Let $\zeta(s)$ and $L(s, \chi)$ be the Riemann zeta function and the Dirichlet L-function respectively. We put

$$
G(s, k)=(-\sqrt{-1})^{k} \sqrt{\pi} \frac{\Gamma(s) \Gamma(s-1 / 2)}{\Gamma(s+k / 2) \Gamma(s-k / 2)} .
$$

(1) When $\chi$ is a non-trivial character, the constant term matrix is given by

$$
M(s, k, \chi)=G(s, k) \frac{1}{p^{s}}\left(\begin{array}{cc}
0 & \frac{L(2 s-1, \bar{\chi})}{L(2 s, \bar{\chi})} \\
(-1)^{k} \frac{L(2 s-1, \chi)}{L(2 s, \chi)}, & 0
\end{array}\right) .
$$

(2) When $\chi$ is a trivial character $\chi_{0}$, the constant term matrix is given by

$$
M\left(s, k, \chi_{0}\right)=G(s, k) \frac{\zeta(2 s-1)}{\zeta(2 s)}\left(\begin{array}{cc}
\frac{p-1}{p^{2 s}-1}, & \frac{1-p^{1-2 s}}{p^{s}\left(1-p^{-2 s}\right)} \\
\frac{1-p^{1-2 s}}{p^{s}\left(1-p^{-2 s}\right)}, & \frac{p-1}{p^{2 s}-1}
\end{array}\right) .
$$

Proof. We apply the double coset decomposition

$$
\Gamma_{\infty} \backslash \sigma_{\kappa}^{-1} \Gamma \sigma_{\mu} / \Gamma_{\infty}=\delta_{\kappa, \mu} \Gamma_{\infty} \cup\left\{\bigcup_{c, d} \Gamma_{\infty}\left(\begin{array}{ll}
* & * \\
c & d
\end{array}\right) \Gamma_{\infty}\right\},
$$

with $c>0, d \bmod c$ and $\left(\begin{array}{l}* \\ c\end{array} \underset{d}{*}\right) \in \sigma_{x}^{-1} \Gamma \sigma_{\mu}$.

Case (i) $\kappa=\mu=\infty$. We have

$$
\begin{aligned}
& a_{\infty \infty \infty}(0 ; y, s)=y^{s}+\int_{0}^{1} \sum_{\left.\substack{* \\
c}) \in \Gamma_{\infty}\right) \in \Gamma_{\infty} \backslash \Gamma} \chi(d)^{-1}\left(\frac{c z+d}{|c z+d|}\right)^{-k} \frac{y^{s}}{|c z+d|^{2 s}} d x \\
& =y^{s}+\sum_{\substack{c=1 \\
\text { plc } \\
d \in(\boldsymbol{Z} / c \mathcal{Z}) \times}}^{\infty} \frac{\chi(d)^{-1}}{c^{2 s}} \int_{-\infty}^{\infty}\left(\frac{z+d / c}{|z+d / c|}\right)^{-k} \frac{y^{s}}{|z+d / c|^{2 s}} d x \\
& =y^{s}+y^{1-s}\left(\sum_{\substack{c=1 \\
\text { ple } \\
d \in(Z / c Z) \times}}^{\infty} \frac{\chi(d)^{-1}}{c^{2 s}}\right) \int_{-\infty}^{\infty} \frac{(x-\sqrt{-1})^{k}}{\left(x^{2}+1\right)^{s+k / 2}} d x .
\end{aligned}
$$

The last integral is equal to 


$$
(-\sqrt{-1})^{k} \sqrt{\pi} \frac{\Gamma(s) \Gamma(s-1 / 2)}{\Gamma(s+k / 2) \Gamma(s-k / 2)} .
$$

Next we consider the last sum. It vanishes for a non-trivial character. For a trivial character, we have

$$
\begin{aligned}
\sum_{c, d} \frac{1}{c^{2 s}} & =\sum_{\substack{c=1 \\
p=c}}^{\infty} \frac{\varphi(c)}{c^{2 s}} \\
& =\left(\prod_{\substack{q: \text { prime } \\
q \neq p}} \sum_{m=0}^{\infty} \frac{\varphi\left(q^{m}\right)}{q^{2 s m}}\right)\left(\sum_{m=1}^{\infty} \frac{\varphi\left(p^{m}\right)}{p^{2 s m}}\right) \\
& =\left(\sum_{\substack{q: \text { prime } \\
q \neq p}} \frac{1-q^{-2 s}}{1-q^{1-2 s}}\right)\left(1-p^{-1}\right) \frac{p^{1-2 s}}{1-p^{1-2 s}} \\
& =\frac{\zeta(2 s-1)}{\zeta(2 s)} \frac{p-1}{p^{2 s-1}} .
\end{aligned}
$$

where $\varphi$ is the Euler function.

Case (ii) $\kappa=\infty$ and $\mu=0$. In this case, we have

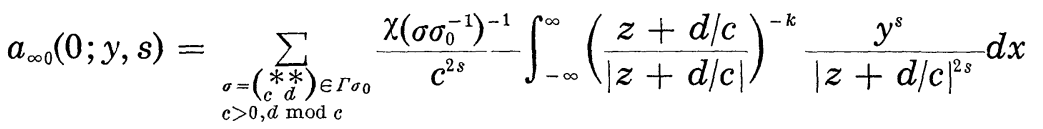

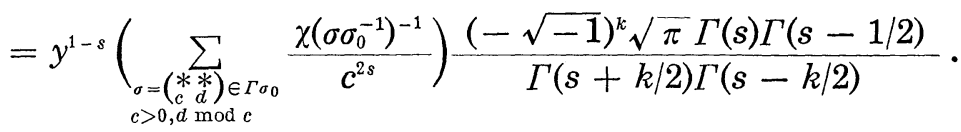

Put

$$
\begin{aligned}
\sigma & =\left(\begin{array}{ll}
* & * \\
c & d
\end{array}\right)=\left(\begin{array}{cc}
* & * \\
m & n
\end{array}\right) \sigma_{0} \\
& =\left(\begin{array}{cc}
* & * \\
n \sqrt{p}-m / \sqrt{p}
\end{array}\right) \quad \text { for }\left(\begin{array}{cc}
* & * \\
m & n
\end{array}\right) \in \Gamma .
\end{aligned}
$$

We see that the condition $d \bmod c$ reduces to $m / p \bmod n$, hence the above sum is equal to

$$
\frac{1}{p^{s}} \sum_{\substack{n=1 \\(p, n)=1}}^{\infty} \frac{\chi(n)^{-1} \varphi(n)}{n^{2 s}}=\frac{1}{p^{s}} \frac{L(2 s-1, \bar{\chi})}{L(2 s, \bar{\chi})} .
$$

In particular, when $\chi$ is a trivial character, it is equal to

$$
\frac{1}{p^{s}} \frac{\zeta(2 s-1)}{\zeta(2 s)} \frac{1-p^{1-2 s}}{1-p^{-2 s}} .
$$

Case (iii) $\kappa=0$ and $\mu=\infty$. By the same way as in the case (ii), we get 


$$
a_{0 \infty}(0 ; y, s)=y^{1-s} \frac{(-1)^{k}}{p^{s}} \frac{L(2 s-1, \chi)}{L(2 s, \chi)} \frac{(-\sqrt{-1})^{k} \sqrt{\pi} \Gamma(s) \Gamma(s-1 / 2)}{\Gamma(s+k / 2) \Gamma(s-k / 2)} .
$$

In particular, when $\chi$ is a trivial character, we get

$$
a_{\infty 0}(0 ; y, s)=a_{0 \infty}(0 ; y, s) \text {. }
$$

Case (iv) $\kappa=\mu=0$. This case is reduced to case (i), so we get

$$
a_{00}(0 ; y, s)=a_{\infty \infty}(0 ; y, s) \text {. }
$$

The cases above complete the proof of Proposition 1.

The Fourier coefficient $a_{\kappa, \mu}(m ; y, s)$ for $m \neq 0$ can also be evaluated by the integral in (1.2) using the Whittaker function $W_{\alpha, \beta}$. In fact, we have

$$
\begin{aligned}
& a_{\kappa, \mu}(m ; y, s)=-(-\sqrt{-1})^{k} \pi^{s} \frac{\operatorname{sgn}(m)|m|^{s-1}}{\Gamma(s+\operatorname{sgn}(m)(k / 2))} \\
& \quad \times W_{k \cdot \operatorname{sgn}(m), 1 / 2-s}(4 \pi|m| y)\left\{\sum_{\substack{\sigma \in \Gamma_{\infty}^{\infty} \sigma_{k}-1 \Gamma \sigma_{\mu} / \Gamma_{\infty} \\
\sigma=\left(\begin{array}{l}
* * * \\
c *
\end{array}\right), c>0, d \bmod c}} \frac{\chi\left(\sigma_{\kappa} \sigma \sigma_{\mu}^{-1}\right)^{-1}}{c^{2 s}} e\left(\frac{m d}{c}\right)\right\} .
\end{aligned}
$$

Proposition 2. The constant term matrix $M(s, k, \chi)$ and Eisenstein series $E_{x}(z, s)$ can be continued meromorphically to the whole plane and satisfy the functional equations

$$
\begin{gathered}
M(s, k, \chi) M(1-s, k, \chi)=I, \\
\left(\begin{array}{l}
E_{\infty}(z, 1-s) \\
E_{0}(z, 1-s)
\end{array}\right)=M(1-s, k, \chi)\left(\begin{array}{l}
E_{\infty}(z, s) \\
E_{0}(z, s)
\end{array}\right) .
\end{gathered}
$$

The above Proposition is a consequence of the general theory of Eisenstein series (cf. [6]), but in our case, we can show (1.6) directly by using the functional equation of the Riemann zeta or the Dirichlet $L$ function.

\section{§2. Dimension formula}

Let $G$ denote $S L(2, R)$. We choose a fundamental domain $\mathscr{D}$ of $\Gamma$ in $S$ such that $\mathscr{D}$ has $\infty$ and 0 as the cusps. We put $\tilde{S}=S \times(R / 2 \pi Z)$. The group $G$ operates on $\tilde{S}$ as

$$
g(z, \phi)=\left(\frac{a z+b}{c z+d}, \phi+\arg (c z+d)\right)
$$

for $(z, \phi) \in \tilde{S}, g=\left(\begin{array}{ll}a & b \\ c & d\end{array}\right) \in G$. We choose $d(z, \phi)=y^{-2} d x d y d \phi$ as the $G$ - 
invariant measure on $\tilde{S}$. It is well known that the ring of $G$-invariant differential operators on $\tilde{S}$ is generated by

$$
\frac{\partial}{\partial \phi} \text { and } \tilde{\Delta}=y^{2}\left(\frac{\partial^{2}}{\partial x^{2}}+\frac{\partial^{2}}{\partial y^{2}}\right)+\frac{5}{4} \frac{\partial^{2}}{\partial \phi^{2}}+y \frac{\partial}{\partial \phi} \frac{\partial}{\partial x} \text {. }
$$

We fix such a fundamental domain $\tilde{D}$ of $\Gamma$ in $\tilde{S}$ as it is projected on $\mathscr{D}$. Let $L^{2}(\tilde{\mathscr{D}}, \chi)$ be the set of all functions on $\tilde{S}$ such that

(i) $f(\sigma(z, \phi))=\chi(\sigma) f(z, \phi)$ for all $\sigma \in \Gamma$,

(ii) $\int_{\tilde{g}}|f(z, \phi)|^{2} d(z, \phi)<\infty$.

We denote by $\mathfrak{M}(k, \lambda, \chi)$ the set of all functions in $L^{2}(\widetilde{\mathscr{D}}, \chi)$ which satisfy the further conditions

(iii) $(\partial / \partial \phi) f(z, \phi)=-\sqrt{-1} k f(z, \phi)$,

(iv) $\tilde{\Delta} f(z, \phi)=\lambda f(z, \phi)$.

It is well known that every eigenspace $\mathfrak{M}(k, \lambda, \chi)$ is finite dimensional and orthogonal to each other.

Now we consider the space of cusp forms of weight 1 . It is shown in [2] that $\mathfrak{M}(1,-3 / 2, \chi) \cong S_{1}(\Gamma, \chi)$. We suppose that $p \geq 3$ in the sequal because $S_{1}(\Gamma, \chi)=\{0\}$ if $p=2$. First we introduce an invariant integral operator on $C^{\infty}(\tilde{S})$ defined by a point-pair invariant kernel

$$
\begin{aligned}
& \omega_{\delta}\left((z, \phi),\left(z^{\prime}, \phi^{\prime}\right)\right) \\
& \quad=\left|\frac{\left(y y^{\prime}\right)^{1 / 2}}{\left(z-\bar{z}^{\prime}\right) / 2 \sqrt{-1}}\right|^{\delta} \frac{\left(y y^{\prime}\right)^{1 / 2}}{\left(z-\bar{z}^{\prime}\right) / 2 \sqrt{-1}} e^{-\sqrt{-1}\left(\phi-\phi^{\prime}\right)}, \quad \delta \in C .
\end{aligned}
$$

For $\operatorname{Re}(\delta)>1$, it is of (a)-(b) type in the sense of Selberg [7]. Put

$$
\left.K_{\delta}\left((z, \phi),\left(z^{\prime}, \phi^{\prime}\right)\right)=\sum_{\sigma \in \Gamma} \chi(\sigma) \omega_{\dot{o}}(z, \phi), \sigma\left(z^{\prime}, \phi^{\prime}\right)\right) .
$$

Then the operator $\omega_{\delta}$ can be written as

$$
\int_{\tilde{\Phi}} K_{\delta}\left((z, \phi),\left(z^{\prime}, \phi^{\prime}\right)\right) f\left(z^{\prime}, \phi^{\prime}\right) d\left(z^{\prime}, \phi^{\prime}\right)
$$

on $L^{2}(\tilde{\mathscr{D}}, \chi)$. By the definition, the operator $K_{\delta}$ vanishes on $\mathfrak{M}(k, \lambda, \chi)$ unless $k=1$. Put $\lambda_{0}=-3 / 2$. Let $-3 / 2>\lambda_{1}>\lambda_{2}>\ldots$ are the set of all eigenvalues of $\tilde{\Delta}$ in $L^{2}(\tilde{\mathscr{D}}, \chi)$ such that $\mathfrak{M}\left(1, \lambda_{i}, \chi\right) \neq\{0\}$. It is known that $\lambda_{i} \rightarrow$ $-\infty$ when $i \rightarrow \infty$. It follows from [7] that every non zero element in $\mathfrak{M}\left(1, \lambda_{i}, \chi\right)$ is an eigenfunction of $K_{\delta}$ and its eigenvalue depends only on the spectrum $\lambda$. In fact, it is easy to see that the eigenvalue is given by 


$$
h_{\delta}(r)=2^{\delta+2} \pi \frac{\Gamma(1 / 2) \Gamma((\delta+1) / 2)}{\Gamma(\delta) \Gamma(\delta / 2+1)} \Gamma(\delta / 2+\sqrt{-1} r) \Gamma(\delta / 2-\sqrt{-1} r)
$$

with $\lambda=-3 / 2-r^{2}$.

Put $E_{x}^{*}(z, \phi, s, 1, \chi)=e^{-\sqrt{-1} \phi} E_{x}(z, s, 1, \chi)$. We also write simply as $E_{\kappa}^{*}(z, \phi, s)=E_{\kappa}^{*}(z, \phi, s, 1, \chi)$. We define

$$
\begin{aligned}
& H_{\delta}^{\kappa}\left((z, \phi),\left(z^{\prime}, \phi^{\prime}\right)\right) \\
& \quad=\frac{1}{4 \pi^{2}} \int_{-\infty}^{\infty} h_{\delta}(r) E_{\kappa}^{*}\left(z, \phi, \frac{1}{2}+\sqrt{-1} r\right) \overline{E_{\kappa}^{*}\left(z^{\prime}, \phi^{\prime}, \frac{1}{2}+\sqrt{-1} r\right)} d r .
\end{aligned}
$$

As is well known, the integral operator on $L^{2}(\widetilde{\mathscr{D}}, \chi)$ defined by the kernel $K_{\delta}^{*}=K_{\delta}-H_{\hat{o}}^{\infty}-H_{\delta}^{0}$ is completely continuous and has all discrete spectra of $K_{\delta}$. So we obtain the following trace formula

$$
\sum_{i=0}^{\infty} h_{\delta}\left(r_{i}\right) \operatorname{dim} \mathfrak{M}\left(1, \lambda_{i}, \chi\right)=\int_{\tilde{\mathscr{D}}} K_{\delta}^{*}((z, \phi),(z, \phi)) d(z, \phi),
$$

where $\lambda_{i}=-3 / 2-r_{i}^{2}$. It was shown by Selberg that the right hand side of (2.3) may be reduced to the sum of components corresponding to conjugacy classes of $\Gamma$. We will compute each term in the next Theorem.

Before that, we set the following definition.

Definition. Let $\left\{P_{\alpha}\right\}$ denote the representatives of primitive hyperbolic conjugacy classes in $\Gamma /\{ \pm I\}$. We define the Selberg type zeta function by

$$
z(\delta, \chi)=\sum_{\alpha=1}^{\infty} \sum_{l=1}^{\infty} \frac{\chi\left(P_{\alpha}^{l}\right) \operatorname{sign}\left(\lambda_{\alpha}^{l}\right) \log N\left(P_{\alpha}\right)}{\left(N\left(P_{\alpha}\right)^{l / 2}-N\left(P_{\alpha}\right)^{-l / 2}\right)\left(N\left(P_{\alpha}\right)^{l / 2}+N\left(P_{\alpha}\right)^{-l / 2}\right)^{\delta}}
$$

where $\lambda_{\alpha}$ is an eigenvalue of $P_{\alpha}$ and $N\left(P_{\alpha}\right)$ is the norm of $P_{\alpha}$.

TheOREM 1. For $\operatorname{Re}(\delta)>1$, the following trace formula holds:

$$
\begin{aligned}
& \sum_{i=0}^{\infty} h_{\delta}\left(r_{i}\right) \operatorname{dim} \mathfrak{M}\left(1, \lambda_{i}, \chi\right) \\
= & \frac{2 \pi^{2}}{3}(p+1)+2^{\delta+2} \pi \frac{\Gamma(1 / 2) \Gamma((\delta+1) / 2)}{\Gamma(\delta / 2+1)} z(\delta, \chi) \\
+ & \frac{8 \pi^{2}}{3 \sqrt{-3}} \frac{a_{p}}{\delta}\left(F\left(1, \frac{\delta}{2} ; \delta+1 ; \frac{1+\sqrt{-3}}{2}\right)-F\left(1, \frac{\delta}{2} ; \delta+1 ; \frac{1-\sqrt{-3}}{2}\right)\right) \\
+ & 4 \pi \frac{\Gamma(1 / 2) \Gamma((\delta+1) / 2)}{\Gamma(\delta / 2+1)}\left(\log \left(\pi^{2} / 4 p^{3}\right)+2^{\delta} \frac{\Gamma((\delta+1) / 2)^{2}}{\Gamma(\delta+1)}\right) \\
- & \frac{1}{\pi} \frac{\Gamma((\delta+1) / 2) \Gamma((\delta+3) / 2)}{\Gamma(\delta / 2+1)^{2}} \int_{-\infty}^{\infty} h_{\delta+1}(r) \frac{\Gamma^{\prime}}{\Gamma}(1+\sqrt{-1} r) d r
\end{aligned}
$$




$$
\begin{aligned}
& -\frac{1}{\pi} \int_{-\infty}^{\infty} h_{\delta}(r) \frac{\Gamma^{\prime}}{\Gamma}(1+\sqrt{-1} r) d r \\
& -\frac{1}{\pi} \int_{-\infty}^{\infty} h_{\delta}(r)\left(\frac{L^{\prime}}{L}(1+2 \sqrt{-1} r, \chi)+\frac{L^{\prime}}{L}(1+2 \sqrt{-1} r, \bar{\chi})\right) d r,
\end{aligned}
$$

where

$$
a_{p}=\left\{\begin{array}{cl}
\chi(c)+\chi\left(c^{\prime}\right) & \text { for } p \equiv 1(\bmod 3) \\
0 & \text { for } p \equiv 2(\bmod 3) \\
-1 & \text { for } p=3
\end{array}\right.
$$

and $c, c^{\prime}$ are two solutions of $x^{2}-x+1 \equiv 0(\bmod p)$.

Proof. Denote by $\{M\}$ the conjugacy class of $M \in \Gamma$. We put

$$
J(M)=\int_{\tilde{\mathscr{D}}} \sum_{\sigma \in\{M\}} \chi(\sigma) \omega_{\hat{o}}((z, \phi), \sigma(z, \phi)) d(z, \phi)
$$

for $M$ which is not a parabolic element. Let $P_{\kappa}$ be the set of all parabolic elements of $\Gamma$ which fix a cusp equivalent to $\kappa$. We put

$$
J(\kappa)=\int_{\tilde{g}}\left\{\sum_{\sigma \in P_{\kappa}} \chi(\sigma) \omega_{\delta}((z, \phi), \sigma(z, \phi))-H_{\delta}^{x}((z, \phi),(z, \phi))\right\} d(z, \phi) .
$$

Then we have, as usual,

$$
\begin{aligned}
& \int_{\tilde{\mathscr{D}}} K_{\delta}^{*}((z, \phi),(z, \phi)) d(z, \phi) \\
& \quad=J(I)+J(-I)+\sum_{\{Q\}} J(Q)+\sum_{\{R\}} J(R)+J(\infty)+J(0),
\end{aligned}
$$

where $Q$, and $R$ denote respectively a hyperbolic element, and an elliptic element in $\Gamma$.

(i) It is clear that

$$
J(I)=J(-I)=\operatorname{vol}(\widetilde{\mathscr{D}})=\frac{\pi^{2}}{3}(p+1) .
$$

(ii) Let $Q$ be a hyperbolic element of $\Gamma$. There exists an element $Q_{0}$ such that $\left\{ \pm Q_{0}^{n} \mid n \in Z\right\}$ is the centralizer of $Q$. Let $\lambda$ and $\lambda_{0}$ be eigenvalues of $Q$ and $Q_{0}$ with $|\lambda|,\left|\lambda_{0}\right|>1$ respectively. Then it is easy to see that

$$
J(Q)=2^{\delta+2} \pi \frac{\Gamma(1 / 2) \Gamma((\delta+1) / 2)}{\Gamma(\delta / 2+1)} \frac{\chi(Q) \operatorname{sign}(\lambda) \log \left|\lambda_{0}\right|}{\left(|\lambda|-|\lambda|^{-1}\right)\left(|\lambda|+|\lambda|^{-1}\right)^{\delta}} .
$$

Therefore we have 


$$
\sum_{\{Q\}} J(Q)=2^{\delta+2} \pi \frac{\Gamma(1 / 2) \Gamma((\delta+1) / 2)}{\Gamma(\delta / 2+1)} z^{\prime}(\delta, \chi)
$$

(iii) Let $R$ be an elliptic element of $\Gamma$ with $\rho$ as the fixed point in $S$. Let $\psi$ be a linear transformation which maps $S$ into a unit circle: $w=\psi(z)=(z-\rho) /(z-\bar{\rho})$. We put $R=\left(\begin{array}{ll}a & b \\ c & d\end{array}\right)$ and $\zeta=c \bar{\rho}+d$. Then $\zeta$ and $\bar{\zeta}$ are eigenvalues of $R$, and $w^{\prime}=\psi(R z)=(\zeta / \bar{\zeta}) w$. By the simple calculation, we have

$$
\begin{array}{rlrl}
J(R) & =\frac{8 \pi \chi(R) \bar{\zeta}}{|\Gamma(R)|} \int_{|w|<1} \frac{\left(1-|w|^{2}\right)^{\hat{j}-1}}{\left|1-\bar{\zeta}^{2} w \bar{w}\right|^{\delta}\left(1-\bar{\zeta}^{2} w \bar{w}\right)} d u d v & & (w=u+\sqrt{-1} v) \\
& =\frac{16 \pi^{2} \chi(R) \bar{\zeta}}{|\Gamma(R)|} \int_{0}^{1} \frac{\left(1-r^{2}\right)^{\delta-1} r}{\left|1-\bar{\zeta}^{2} r^{2}\right|^{\delta}\left(1-\bar{\zeta}^{2} r^{2}\right)} d r & & \left(w=r e^{\sqrt{-1} \theta}\right) \\
& =\frac{8 \pi^{2} \chi(R)}{|\Gamma(R)|} \frac{1}{\zeta-\bar{\zeta}} \frac{1}{\delta} F\left(1, \frac{\delta}{2} ; \delta+1 ; 1+\zeta^{2}\right), &
\end{array}
$$

where $F$ is the hypergeometric function and $\Gamma(R)$ is the centralizer of $R$ in $\Gamma$. Now we compute the sums $\sum_{\text {order } 4} J(R), \sum_{\text {order } 3 \text { or } 6} J(R)$. Put $\gamma_{c}=$ $\left(\begin{array}{ll}1 & 0 \\ c & 1\end{array}\right) \in S L(2, Z)$ for $c \bmod p$. First we consider the case of order 4 . Suppose that $p \equiv 1(\bmod 4)$, and let $c, c^{\prime}$ be two solutions of $x^{2}+1 \equiv 0(\bmod$ $p$ ). There exist four conjugacy classes of elliptic elements of order 4 in $\Gamma$, and they are represented by $\pm \gamma_{c} \tau \gamma_{c}^{-1}$ and $\pm \gamma_{c^{\prime}} \tau \gamma_{c^{\prime}}^{-1}$ where $\tau=\left(\begin{array}{rr}0 & 1 \\ -1 & 0\end{array}\right)$, Therefore we have

$$
\begin{aligned}
\sum_{\text {order } 4} J(R) & =2\left(J\left(\gamma_{c} \tau \gamma_{c}^{-1}\right)+J\left(\gamma_{c^{\prime}} \tau \gamma_{c^{\prime}}^{-1}\right)\right) \\
& =-\frac{2 \pi^{2} \sqrt{-1}}{\delta} F\left(1, \frac{\delta}{2} ; \delta+1 ; 0\right)\left(\chi(c)+\chi\left(c^{\prime}\right)\right) \\
& =0
\end{aligned}
$$

because $\chi$ is an odd character. When $p \equiv 3(\bmod 4)$, there are no elliptic elements of order 4. Next we consider the case of order 3 or 6 . Suppose that $p \equiv 1(\bmod 3)$, and let $c$ and $c^{\prime}$ be two solutions of $x^{2}-x+1 \equiv 0$ $(\bmod p)$. Then there exist eight conjugacy classes of elliptic elements of order 3 or 6 , and are represented by $\pm \gamma_{c} \tau_{j} \gamma_{c}^{-1}$, and $\pm \gamma_{c^{\prime}} \tau_{j} \gamma_{c^{\prime}}^{-1}(j=1,2)$ where $\tau_{1}=\left(\begin{array}{rr}0 & 1 \\ -1 & -1\end{array}\right)$ and $\tau_{2}=\left(\begin{array}{rr}-1 & -1 \\ 1 & 0\end{array}\right)$. On the other hand, when $p=$ 3 , there are four conjugacy classes of elliptic elements of order 3 or 6 , and are represented by $\pm \gamma_{2} \tau_{j} \gamma_{2}^{-1}$. Hence we have 


$$
\begin{aligned}
\sum_{\text {order } 3 \text { or } 6} J(R) & =2 \sum_{j=1}^{2}\left\{J\left(\gamma_{c} \tau_{j} \gamma_{c}^{-1}\right)+J\left(\gamma_{c^{\prime}} \tau_{j} \gamma_{c^{\prime}}^{-1}\right)\right\} \\
= & \frac{8 \pi^{2}}{3 \sqrt{-3}} \frac{1}{\delta}\left(F\left(1, \frac{\delta}{2} ; \delta+1 ; \frac{1+\sqrt{-3}}{2}\right)\right. \\
& \left.-F\left(1, \frac{\delta}{2} ; \delta+1 ; \frac{1-\sqrt{-3}}{2}\right)\right)\left(\chi(c)+\chi\left(c^{\prime}\right)\right)
\end{aligned}
$$

for $p \equiv 1(\bmod 3)$, and

$$
\begin{aligned}
& \sum_{\text {order } 3 \text { or } 6} J(R)=2\left(J\left(\gamma_{2} \tau_{1} \gamma_{2}^{-1}\right)+J\left(\gamma_{2} \tau_{2} \gamma_{2}^{-1}\right)\right) \\
&=\frac{-8 \pi^{2}}{3 \sqrt{-3}} \frac{1}{\delta}\left(F\left(1, \frac{\delta}{2} ; \delta+1 ; \frac{1+\sqrt{-3}}{2}\right)\right. \\
&\left.-F\left(1, \frac{\delta}{2} ; \delta+1 ; \frac{1-\sqrt{-3}}{2}\right)\right)
\end{aligned}
$$

for $p=3$. When $p \equiv 2(\bmod 3)$, there are no elliptic elements of order 3 or 6 .

(iv) We follow the method of [6] to compute $J(\kappa)$. Let $\mathscr{D}_{Y}^{\prime}$ be the subdomain of $\mathscr{D}$ consisting of all points $z \in \mathscr{D}$ such that $y>Y$. Put $\mathscr{D}_{Y}$ $=\mathscr{D} \backslash\left\{\mathscr{D}_{Y}^{\prime} \cup \sigma_{0} \mathscr{D}_{Y}^{\prime}\right\}$ and $\widetilde{\mathscr{D}}_{Y}=\left\{(z, \phi) \in \widetilde{\mathscr{D}} \mid z \in \mathscr{D}_{Y}\right\}$. We also put $T=\left(\begin{array}{ll}1 & 1 \\ 0 & 1\end{array}\right)$. Every element of $P_{\kappa}$ is conjugate to $\pm \sigma_{x} T^{m} \sigma_{\kappa}^{-1}$ for some non zero integer $m$, and every $\pm \sigma_{k} T^{m} \sigma_{k}^{-1}$ is not conjugate to each other. Therefore, we have

$$
\begin{aligned}
& \int_{\tilde{\mathscr{T}}_{Y}} \sum_{\sigma \in P_{\kappa}} \chi(\sigma) \omega_{\delta}((z, \phi), \sigma(z, \phi)) d(z, \phi) \\
& \quad=2 \sum_{m \neq 0} \int_{\tilde{\mathscr{T}}_{Y}} \sum_{r \in \Gamma_{\kappa} \backslash \Gamma} \chi\left(\gamma^{-1} \sigma_{\kappa} T^{m} \sigma_{\kappa}^{-1} \gamma\right) \omega_{\delta}\left((z, \phi), \gamma^{-1} \sigma_{\kappa} T^{m} \sigma_{\kappa}^{-1} \gamma(z, \phi)\right) d(z, \phi) \\
& \quad=2 \sum_{m \neq 0} \int_{\tilde{\mathscr{F}}} \omega_{\delta}\left((z, \phi), T^{m}(z, \phi)\right) d(z, \phi) \\
& \quad=2 \pi \sum_{m \neq 0} \int_{\mathscr{F}} \omega_{\delta}\left((z, 0), T^{m}(z, 0)\right) \frac{d x d y}{y^{2}},
\end{aligned}
$$

where $\tilde{\mathscr{F}}=\cup_{r} \gamma \sigma_{\kappa}^{-1} \widetilde{\mathscr{D}}_{Y},\left(\gamma \in \Gamma_{\infty} \mid \sigma_{\kappa}^{-1} \Gamma \sigma_{\kappa}\right)$ and $\mathscr{F}$ is the projection of $\tilde{\mathscr{F}}$ onto $S$. It is easy to see that the last sum of the integral on $\{z \in S \mid 0 \leq x \leq 1$, $y \leq Y\} \backslash \mathscr{F}$ is $o(1)$ as $Y \rightarrow \infty$. Therefore, (2.6) is equal to

$$
\begin{array}{r}
-2 \pi \sqrt{-1} \sum_{m \neq 0} \int_{0}^{Y} \frac{(m / 2 y)+\sqrt{-1}}{\left((m / 2 y)^{2}+1\right)^{\delta / 2+1}} \frac{d y}{y^{2}}+o(1) \\
=4 \pi \sum_{m=1}^{\infty} \int_{0}^{Y} \frac{1}{\left((m / 2 y)^{2}+1\right)^{\delta / 2+1}} \frac{d y}{y^{2}}+o(1) .
\end{array}
$$


Now we apply the Euler-MacLaurin summation formula as described in [6]. As a result, (2.6) becomes

$$
\begin{aligned}
4 \pi & \frac{\Gamma(1 / 2) \Gamma((\delta+1) / 2)}{\Gamma(\delta / 2+1)}(\log 2 Y+C)+8 \pi \int_{0}^{\infty} \frac{\log t}{\left(t^{2}+1\right)^{\delta / 2+1}} d t+o(1) \\
= & 4 \pi \frac{\Gamma(1 / 2) \Gamma((\delta+1) / 2)}{\Gamma(\delta / 2+1)}\left(\log (Y / 2)+2^{\delta-1} \frac{\Gamma((\delta+1) / 2)^{2}}{\Gamma(\delta+1)}\right) \\
& -\frac{1}{2 \pi} \frac{\Gamma((\delta+1) / 2) \Gamma((\delta+3) / 2)}{\Gamma(\delta / 2+1)^{2}} \int_{-\infty}^{\infty} h_{\delta+1}(r) \frac{\Gamma^{\prime}}{\Gamma}(1+\sqrt{-1} r) d r+o(1)
\end{aligned}
$$

as $Y \rightarrow \infty$, where $C$ is the Euler constant.

The Eisenstein part is evaluated most elegantly by using the MaaßSelberg relation. We define the compact part of $E_{\kappa}^{*}(z, \phi, s)$ by

$$
\begin{aligned}
& E_{\kappa}^{* Y}(z, \phi, s) \\
& \quad= \begin{cases}E_{\kappa}^{*}(z, \phi, s)-e^{-\sqrt{-1} \phi} j_{1}\left(\sigma_{\mu}^{-1}, z\right)^{-1} a_{\kappa, \mu}\left(0 ; \operatorname{Im}\left(\sigma_{\mu}^{-1} z\right), s\right) & \text { if } z \in \sigma_{\mu} \mathscr{D}_{Y}^{\prime}, \\
E_{\kappa}^{*}(z, \phi, s) & \text { otherwise }\end{cases}
\end{aligned}
$$

Then the Maaß-Selberg relation states that

$$
\frac{1}{\pi}\left(E_{\kappa}^{* Y}(z, \phi, s), E_{\kappa}^{* Y}\left(z, \phi, \bar{s}^{\prime}\right)\right)=\frac{Y^{s+s^{\prime}-1}-m_{\kappa, \mu}(s, 1, \chi) \overline{m_{\kappa, \mu}\left(\bar{s}^{\prime}, 1, \chi\right)} Y^{-s-s^{\prime}+1}}{s+s^{\prime}-1},
$$

$(\kappa \neq \mu)$. Hence, putting $s=t+\sqrt{-1} r$, we have

$$
\begin{aligned}
\int_{\tilde{\mathscr{D}}_{Y}} H_{\delta}^{\kappa}((z, \phi),(z, \phi)) d(z, \phi) \\
=\frac{1}{4 \pi^{2}} \lim _{t \rightarrow 1 / 2} \int_{\tilde{\mathscr{D}}} \int_{-\infty}^{\infty} h_{\delta}(r) E_{\kappa}^{* Y}(z, \phi, s) \overline{E_{\kappa}^{* Y}(z, \phi, s)} d r d(z, \phi)+o(1) \\
=\frac{1}{4 \pi} \lim _{t \rightarrow 1 / 2} \int_{-\infty}^{\infty} h_{\delta}(r) \frac{Y^{2 t-1}-m_{\kappa, \mu}(s, 1, \chi) \overline{m_{x, \mu}(s, 1, \chi)} Y^{1-2 t}}{2 t-1} d r+o(1) \\
=4 \pi \frac{\Gamma(1 / 2) \Gamma((\delta+1) / 2)}{\Gamma(\delta / 2+1)} \log Y \\
\quad-\frac{1}{4 \pi} \int_{-\infty}^{\infty} h_{\delta}(r) \frac{m_{\kappa, \mu}^{\prime}}{m_{\kappa, \mu}}\left(\frac{1}{2}+\sqrt{-1} r, 1, \chi\right) d r+o(1),
\end{aligned}
$$

$(\kappa \neq \mu)$. We used (1.6) in the above computation. We have

$$
\begin{aligned}
& \frac{m_{\infty, 0}^{\prime}}{m_{\infty, 0}}\left(\frac{1}{2}+\sqrt{-1} r, 1, \chi\right)=\frac{m_{0, \infty}^{\prime}}{m_{0, \infty}}\left(\frac{1}{2}+\sqrt{-1} r, 1, \bar{\chi}\right) \\
& \quad=\log \left(\pi^{2} / p^{3}\right)-\left(\frac{\Gamma^{\prime}}{\Gamma}(1+\sqrt{-1} r)+\frac{\Gamma^{\prime}}{\Gamma}(1-\sqrt{-1} r)\right)
\end{aligned}
$$




$$
-2\left(\frac{L^{\prime}}{L}(1+2 \sqrt{-1} r, \chi)+\frac{L^{\prime}}{L}(1-2 \sqrt{-1} r, \bar{\chi})\right)
$$

by (1.3) and the log derivative of the functional equation of $L$. As $h_{\delta}(r)$ is even, it follows from (2.7)-(2.9) that

$$
\begin{aligned}
J(\kappa)= & \lim _{Y \rightarrow \infty} \int_{\tilde{\mathscr{D}}_{Y}}\left\{\sum_{\sigma \in P_{\boldsymbol{\kappa}}} \chi(\sigma) \omega_{\delta}((z, \phi), \sigma(z, \phi))-H_{\delta}^{\kappa}((z, \phi),(z, \phi))\right\} d(z, \phi) \\
= & 2 \pi \frac{\Gamma(1 / 2) \Gamma((\delta+1) / 2)}{\Gamma^{\prime}(\delta / 2+1)}\left(\log \left(\pi^{2} / 4 p^{3}\right)+2^{\delta} \frac{\Gamma((\delta+1) / 2)^{2}}{\Gamma(\delta+1)}\right) \\
& -\frac{1}{2 \pi} \frac{\Gamma((\delta+1) / 2) \Gamma((\delta+3) / 2)}{\Gamma(\delta / 2+1)^{2}} \int_{-\infty}^{\infty} h_{\delta+1}(r) \frac{\Gamma^{\prime}}{\Gamma}(1+\sqrt{-1} r) d r \\
& -\frac{1}{2 \pi} \int_{-\infty}^{\infty} h_{\delta}(r) \frac{\Gamma^{\prime}}{\Gamma}(1+\sqrt{-1} r) d r \\
& -\frac{1}{2 \pi} \int_{-\infty}^{\infty} h_{\delta}(r)\left(\frac{L^{\prime}}{L}(1+2 \sqrt{-1} r, \chi)+\frac{L^{\prime}}{L}(1+2 \sqrt{-1} r, \bar{\chi})\right) d r .
\end{aligned}
$$

Summing up the above cases, we get Theorem 1 .

Corollary. The Selberg type zeta function $z(\delta, \chi)$ can be continued meromorphically to the whole plane.

Proof. Firstly we consider an analytic continuation of the following series

$$
\sum_{i=0}^{\infty} \Gamma\left(\frac{\delta}{2}+\sqrt{-1} r_{i}\right) \Gamma\left(\frac{\delta}{2}-\sqrt{-1} r_{i}\right) \operatorname{dim} \mathfrak{M}\left(1, \lambda_{i}, \chi\right)
$$

By the theory of trace formula (c.f. [7]), the series above is convergent absolutely and uniformly on any compact set in $\operatorname{Re}(\delta)>1$. Hence (2.11) is a holomorphic function in this region. We put $A=\left\{2\left(m \pm \sqrt{-1} r_{i}\right) \mid m \in Z\right.$, $m \leq 0, i=0,1,2, \cdots\}$. Let $n$ be any positive integer. For $\operatorname{Re}(\delta)>2-2 n$ and $\delta \notin A,(2.11)$ is equal to

$$
\sum_{i=0}^{\infty} \Gamma\left(\frac{\delta}{2}+n+\sqrt{-1} r_{i}\right) \Gamma\left(\frac{\delta}{2}+n-\sqrt{-1} r_{i}\right) \frac{\operatorname{dim} \mathfrak{M}\left(1, \lambda_{i}, \chi\right)}{\prod_{\substack{n=0 \\ j=0}\left((\delta / 2+j)^{2}+r_{i}^{2}\right)}}
$$

Since $\operatorname{Re}(\delta / 2+n)>1$, and $\lim _{i \rightarrow \infty}\left|r_{i}\right|=\infty$, (2.12) is convergent absolutely and uniformly on any compact subset not containing any points of $A$. Hence we can define an analytic continuation of (2.11) to the whole plane.

The terms in the right hand side of (2.5) which are expressed by gamma and hypergeometric functions can be continued meromorphically 
to the whole plane.

Finally we consider the integral

$$
g(\delta)=\int_{-\sqrt{-1} \infty}^{\sqrt{-1} \infty} \Gamma\left(\frac{\delta}{2}+t\right) \Gamma\left(\frac{\delta}{2}-t\right) f(t) d t \quad \text { for } \operatorname{Re}(\delta)>1,
$$

where $f(t)=\left(\Gamma^{\prime} / \Gamma\right)(1+t)$ or $\left(L^{\prime} / L\right)(1+2 t, \chi)$. It is well known that

$$
\frac{\Gamma^{\prime}}{\Gamma}(1+t)=O(\log |t|) \text { and } \frac{L^{\prime}}{L}(1+2 t, \chi)=O(\log 2 p(1+|t|))
$$

for $t \in \sqrt{-1} R$. Then, $g(\delta)$ is a holomorphic function in $\operatorname{Re}(\delta)>0$. In order to continue to the region $\operatorname{Re}(\delta) \leq 0$, we take the point $\delta_{0}$ on $\sqrt{-1} \mathbb{R}$. Let $\mathscr{C}$ be a path described in the figure satisfying that $f(t)$ has no poles in the semi-disks $D_{1}$ and $D_{2}$ surrounded by $\mathscr{C}$ and $\sqrt{-1} R$.

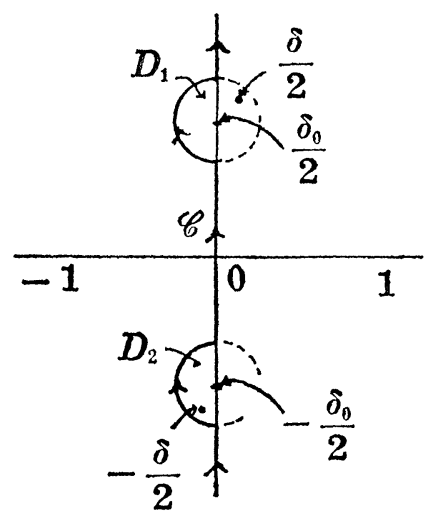

When $\delta_{0}=0$, we take $D_{1}=D_{2}$. Let $\delta$ be very close to $\delta_{0}$ such that $-\delta / 2 \in D_{2}$. It follows from the residue theorem that

$$
g(\delta)=\int_{\mathscr{G}} \Gamma\left(\frac{\delta}{2}+t\right) \Gamma\left(\frac{\delta}{2}-t\right) f(t) d t+2 \pi \sqrt{-1} \Gamma(\delta) f\left(-\frac{\delta}{2}\right) \operatorname{Res}_{t=0} \Gamma(t) .
$$

As the integral above is convergent for any $\delta / 2$ in $D_{1}, g(\delta)$ is continued to the imaginary axis. Let $\delta / 2$ be in the interior of $D_{1}$. Then, $g(\delta)$ can be expressed as

$$
\begin{aligned}
& \int_{-\sqrt{-1} \infty}^{\sqrt{-1} \infty} \Gamma\left(\frac{\delta}{2}+t\right) \Gamma\left(\frac{\delta}{2}-t\right) f(t) d t \\
& \quad+2 \pi \sqrt{-1} \Gamma(\delta)\left(f\left(-\frac{\delta}{2}\right)+f\left(\frac{\delta}{2}\right)\right) \operatorname{Res}_{t=0} \Gamma(t) .
\end{aligned}
$$


The integral above converges in $-2<\operatorname{Re}(\delta)<0$ and defines a holomorphic function in that region. Hence (2.14) gives an analytic continuation of $g(\delta)$ to $-2<\operatorname{Re}(\delta)<0$. By the same way, for $\delta$ in a small neighbourhood of $-2+\delta_{0}\left(\delta_{0} \in \sqrt{-1} R\right)$, we have

$$
\begin{aligned}
g(\delta)= & \int_{\mathscr{8}} \Gamma\left(\frac{\delta}{2}+t\right) \Gamma\left(\frac{\delta}{2}-t\right) f(t) d t \\
& +2 \pi \sqrt{-1} \Gamma(\delta+1) f\left(-\left(\frac{\delta}{2}+1\right)\right) \operatorname{Res}_{t=1}^{\operatorname{es}} \Gamma(t) \\
& +2 \pi \sqrt{-1} \Gamma(\delta)\left(f\left(-\frac{\delta}{2}\right)+f\left(\frac{\delta}{2}\right)\right) \operatorname{Res}_{t=0} \Gamma(t) .
\end{aligned}
$$

And for $-4<\operatorname{Re}(\delta)<-2, g(\delta)$ can be expressed as

$$
\begin{aligned}
& \int_{-\sqrt{-1} \infty}^{\sqrt{-1} \infty} \Gamma\left(\frac{\delta}{2}+t\right) \Gamma\left(\frac{\delta}{2}-t\right) f(t) d t \\
& \quad+2 \pi \sqrt{-1} \Gamma(\delta+1)\left(f\left(-\left(\frac{\delta}{2}+1\right)\right)+f\left(\frac{\delta}{2}+1\right)\right) \operatorname{Res}_{t=-1}^{\operatorname{Res}} \Gamma(t) \\
& \quad+2 \pi \sqrt{-1} \Gamma(\delta)\left(f\left(-\frac{\delta}{2}\right)+f\left(\frac{\delta}{2}\right)\right) \operatorname{Res}_{t=0}^{\operatorname{Ri}} \Gamma(t)
\end{aligned}
$$

Repeating this procedure, $g(\delta)$ can be continued meromorphically to the whole plane. So we get an analytic continuation of $z(\delta, \chi)$.

THeOREM 2. It holds that

$$
\operatorname{dim} S_{1}(\Gamma, \chi)=\frac{1}{4} \operatorname{Res}_{\delta=0} z(\delta, \chi)
$$

Proof. We take the residues of both sides of (2.5) at $\delta=0$. The residue of the left hand side is $16 \pi^{2} \operatorname{dim} S_{1}(\Gamma, \chi)$. In the right hand side of (2.5), the term from elliptic conjugate classes vanishes, because $F(1,0 ; 1 ; z)$ $=1$. The other terms except $z(\delta, \chi)$ turn out to be holomorphic at $\delta=0$. Hence we get Theorem 2 .

\section{REFERENCES}

[1] D. A. Hejhal, The Selberg trace formula for $\operatorname{PSL}(2, R)$ Vol. 2, Lecture Notes in Math., Springer, no. 1001, (1983).

[2] T. Hiramatsu, On some dimension formula for automorphic forms of weight one II, Nagoya Math. J., 105 (1987), 169-186.

[3] T. Hiramatsu and S. Akiyama, On some dimension formula for automorphic forms of weight one III, Nagoya Math. J., 111 (1988), 157-163.

[4] T. Hiramatsu, A formula for the dimension of spaces of cusp forms of weight 1 , 
Advanced Studies in Pure Math., 15, Automorphic forms and Geometry of arithmetic varieties, ed. by K. Hashimoto and Y. Namikawa, Kinokuniya-Academic Press, to appear.

[5] H. Ishikawa, On the trace formula for Hecke operators, J. Fac. Sci. Univ. Tokyo, Sec. IA, 20, no. 2, (1973), 217-238.

[6] T. Kubota, Elementary theory of Eisenstein series, Kodansha and John Wiley, Tokyo-New York, 1973.

[7] A. Selberg, Harmonic analysis and discontinuous groups on weakly symmetric Riemannian spaces with applications to Dirichlet series, J. Indian Math. Soc., 20 (1956), 47-87.

[8] J.-P. Serre, Modular forms of weight one and Galois representations, Algebraic Number Fields, ed. by A. Fröhlich Academic Press (1977), 193-268.

Y. Tanigawa

Department of Mathematics

Faculty of Science

Nagoya University

Chikusa-ku, Nagoya 464

Japan

H. Ishikawa

Department of Mathematics

College of Liberal Arts and Sciences

Okayama University

Tsushima, Okayama 700

Japan 